\title{
Obras didácticas de matemática contextualizadas al Pueblo Indígena Bribri de Costa Rica, a partir de estudios etnomatemáticos ${ }^{1}$
}

\author{
Works contextualized teaching mathematics to Indigenous \\ Bribri of Costa Rica, from studies ethnomathematicians
}

Funciona contextualizado o ensino da matemática para Bribri

Indígena de Costa Rica, a partir de estudos ethnomathematicians

Recibido: mayo de 2013 Aceptado: agosto de 2013
Ana Patricia Vásquez Hernández ${ }^{2}$

\section{Resumen}

El presente proyecto pionero en Costa Rica, se enmarca dentro del campo de la educación matemática de los pueblos originarios. Su objetivo es desarrollar capacidades en los docentes de matemática del Pueblo Indígena Bribri, para la confección colectiva de obras didácticas de matemática contextualizadas y validadas, a través de estudios etnomatemáticos. Lo anterior fortalece su identidad y el respeto por el derecho indígena a un sistema de educativo intercultural. Este proyecto será ejecutado por la Universidad Nacional, Campus Sarapiquí, y por docentes de matemática indígenas de la Dirección Regional de Educación SuLá en Talamanca, a partir del año 2014.

Palabras clave: Libros de texto; Lengua materna; Alumno; Diversidad; Cultura-Religión; Etnomatemática; Proyecto educativo.

\begin{abstract}
This pioneering project in Costa Rica, is part of the field of mathematics education of native peoples. It aims to develop skills in mathematics teachers of Indigenous Bribri, for making collective contextualized mathematics didactic works and validated through studies ethnomathematicians. This strengthens their identity and respect for the indigenous right to intercultural educational system. This project will be implemented by the National University Campus Sarapiqui, and indigenous mathematics teachers of the Regional Directorate of Education Sula in Talamanca, from 2014.
\end{abstract}

Key words: Textbooks, Mother tongue, Student, Diversity, Culture, Religion, Ethnomathematics, educational project.

\section{Resumo}

Este projecto pioneiro em Costa Rica, faz parte do campo da educação matemática dos povos nativos. O objetivo é desenvolver habilidades de professores de matemática de Bribri Indígena, para fazer matemática contextualizadas didáticos obras coletivas e validadas através de estudos

1 Artículo de Investigación.

2 Universidad Nacional de Costa Rica. Contacto: patrimate76@gmail.com 
ethnomathematicians. Isso reforça a sua identidade e respeito ao direito indígena para o sistema educativo intercultural. Este projecto será implementado pelo National University Campus Sarapiqui e professores de matemática indígenas da Direcção Regional de Educação de Sula em Talamanca, a partir de 2014.

Palavras chave: Livros didáticos, língua mãe, Estudante, Diversidade, Cultura, Religião, Etnomatemática, projeto educacional.

\section{Introducción}

El presente proyecto se enmarca dentro del campo estratégico de la educación y el desarrollo integral, cuyas temáticas concordantes son: pueblos originarios, sectores poco favorecidos por la sociedad y educación para una ciudadanía democrática.

Su objetivo es desarrollar capacidades académicas para la confección colectiva de obras didácticas de matemática para el III Ciclo de la Educación General Básica contextualizadas al Pueblo Indígena Bribri validadas por la comunidad educativa, para el fortalecimiento de la identidad cultural y el respeto por el derecho indígena a un sistema de educativo intercultural.

El proyecto ha sido consultado y formulado por la autora de la presente iniciativa, quien funge como responsable del mismo. Sin embargo, será ejecutado por un equipo de trabajo que consiste en dos docentes de matemática del Campus Sarapiquí de la UNA, los docentes de matemática del Territorio Indígena Talamanca Bribri, un asesor pedagógico de la Dirección Regional de Educación del MEP SuLá de Talamanca, estudiantes talentosos de los Liceos Rurales, líderes comunales con conocimientos culturales. Es apoyado por la Escuela de Sociología de la UNA, el Programa de Enseñanza de las Lenguas Indígenas del Departamento de Educación Intercultural del Ministerio de Educación Pública, la Asociación de Desarrollo del Territorio Talamanca Bribri y el Comité Técnico Local de Educación Intercultural de Talamanca (CATLEI).

Esta iniciativa se considera como un proyecto de impacto para la educación matemática de los Territorio Indígenas Bribris, ya que empoderará a los docentes de u conocimiento matemático para integrarlo a la educación formal, rescata la importancia de la generación de materiales de trabajo contextualizados, con lo cual se estaría abriendo una brecha de cambios positivos, importantes y fundamentales para dar respuesta concreta y tangible a la nueva Política Educativa promulgada en el año 2008 (Garnier, 2008) donde se precisa la visión de una educación costarricense dentro de un marco de respeto por la diversidad cultural y étnica.

Por su parte, impacta también el reforzamiento de la etnomatemática en Costa Rica, no solamente como una línea de investigación, sino como eje fundamental de la recuperación de saberes matemáticos para trabajar a favor de la supresión del discurso estandarizado de educación, e iniciar mediante un dialogo respetuoso con la comunidad educativa, la construcción de obras didácticas pioneras en el país, que integre su propio conocimiento y favorezca una educación matemática más feliz para sus estudiantes de secundaria. Quizás con esta iniciativa, se reduzca la aversión a la matemática.

\section{Marco teórico}

Según se indica en fecha 11 de junio de 2013 en la carta DDC-DEI-451-2013 del Programa de Enseñanza de las lenguas Indígenas del Departamento de Educación Intercultural del Ministerio de Educación Pública, en Costa Rica no existen textos, ni ningún otros tipo de obra didáctica contextualizada para la enseñanza de las asignaturas básicas de la educación secundaria de los pueblos indígenas. Existen algunos materiales para primaria, con elaboración muy antigua y que circulan mimeografiados porque carecen de edición y publicación. En América Latina, se reconoce la existencia de trabajos importantes de contextualización de contenidos de matemática 
a entornos específicos, sin embargo a la fecha, se carece de información detallada de los mismos. Próximamente se socializará el proyecto en la Red Latinoamericana de Etnomatemática, para establecer vinculación con proyectos análogos.

Dentro del marco teórico, en el que se desarrolla del presente proyecto se muestra:

a. Obra didáctica de matemática (Definición de la autora): producto intelectual llámese libro, o folletos de matemática dirigido a educandos, que contempla un método para enseñar la disciplina.

b. Contextualización (Definición de la autora): es diseñar o mejorar ejercicios y problemas de matemática en el contexto del Pueblo Indígena Bribri.

c. Etnomatemática: según D`Ambrosio (2008) la etnomatemática es el estudio que se hace en una cultura étnica diferente o particular. En dicha investigación estarán implicados matemáticos, quienes toman sus propias decisiones, también aquellos miembros de la cultura que han experimentado matemática y su aplicación en su educación particular. Se puede decir que las investigaciones etnomatemáticas no son un estudio matemático específicamente, es más bien una interdisciplinariedad de lo matemático, antropológico e histórico en una cultura específica, tratando de describir el mundo matemático que supone procesos de contar, clasificar, ordenar, calcular, medir, organizar el espacio y el tiempo, estimar e inferir.

d. III Ciclo de la Educación General Básica: consiste en los primeros tres años de la secundaria, es decir los niveles de sétimo, octavo y noveno año.

e. Observación participante: Según Greenwood (2000) es la investigación que se basa en vivir con (o cerca de) un grupo de informantes durante un periodo extendido de tiempo, durante el cual se mantienen conversaciones largas con ellos y se participa en algún grado en la vida local. f. Investigación-acción: Según Greenwood (2000) es un grupo de prácticas multidisciplinarias orientadas a una estructura de compromiso intelectual y ético. Se desarrolla mediante la colaboración de un investigador profesional y los dueños del problema en una organización local, una comunidad o un grupo. El trabajo es colaborativo, lo dirige la comunidad y no el investigador profesional. Los conocimientos del experto son importantes, pero los conocimientos locales se consideran esenciales.

\section{Metodología}

Su metodología en general es la observación participante y la investigación acción basada en Greenwood, D. (2000). El abordaje metodológico del presente proyecto se enfoca en tres etapas a saber: I Etapa de conocimientos previos, II Etapa de abordaje y III Etapa de evaluación y socialización de resultados. Este proyecto se sustenta en la metodología que aporta Grenier (1999) sobre las investigaciones relacionadas con el conocimiento indígena.

Su trabajo es colaborativo y consultivo con los involucrados en el sistema educativo del Territorio Indígena Bribri, con el liderazgo en todos los procesos de los miembros del grupo y las decisiones en el formato y diseño de las obras, serán ajustadas a las necesidades que plantee el equipo de trabajo del territorio.

El papel de la proponente de este proyecto es el de ser mediadora y facilitadora, para la confección de obras didácticas de matemática contextualizadas, con diseño de arte y comunicación visual óptimo, con traducciones a la lengua materna y ajustados a los nuevos Programas de Estudio de Matemática.

\section{Conclusiones}

El presente proyecto hace un llamado a reconocer, el trabajo vinculante que implica un estudio etnomatemático, no solamente como una investigación interesante de observación participante $\mathrm{y}$ 
de investigación acción; sino como un medio para reconocer el pensamiento matemático de pueblos originarios, dar valor a sus conocimientos ancestrales, contextualizar y vincular contenidos del currículo escolar, dotar de libros escolares especializados, fortalecer la propuesta del Ministerio de Educación Pública de Costa Rica y contar con una identidad matemática costarricense.

\section{Referencias}

Garnier, L. (2008). El centro educativo de calidad como eje de la educación costarricense. Costa Rica: Editorial Ministerio de Educación Pública.

Gavarrette, M. Vásquez, A. (2005). Etnomatemática en el Territorio Indígena Talamanca Bribri. Tesis de Licenciatura en la Enseñanza de la Matemática. Costa Rica: Universidad Nacional.
González, A. González, F. (2000). La casa cósmica talamanqueña y sus simbolismos. Costa Rica: Editorial Universidad Nacional Estatal a Distancia.

Greenwood, D. (2000). De la observación a la investigación acción participativa: una visión crítica de las prácticas antropológicas. Revista de antropología Social de Cornell University. 9: 27-49.

Grenier, L. (1999). Conocimiento indígena: guía para el investigador. Costa Rica: Editorial Instituto Tecnológico de Costa Rica y el Centro Internacional Canadiense de Investigadores para el Desarrollo.

MEP. (2009). Lo propio, lo nuestro, lo de todos. Educación e Interculturalidad. Costa Rica: Editorial Ministerio de Educación Pública. 